2. Centre For Teaching And Research In Disaster Medicine And Traumatology, Linköping University, Linköping/Sweden

3. Centre For Teaching And Research In Disaster Medicine And Traumatology, And Department Of Clinical And Experimental Medicine, Linköping University, Linköping/Sweden

Study/Objective: In this study, we aimed to design a questionnaire battery for course and simulation exercise evaluation, and pilot-test the battery by evaluating a course on Advanced Prehospital Trauma Care (APTC).

Background: Many course evaluations suffer from simplistic metrics, such as whether the course participants "enjoyed" the course. In contrast, the current study sought to measure (self-estimated) pre- and post-course knowledge, relevant to specific learning objectives, as well as questions pertaining to specific factors of the simulation exercises used in the course (eg, fidelity/realism, learning objective fit, transferability of tools/procedures, usefulness, among others) were selected based on simulation theory and simulation-based training literature. Methods: Data were collected during a course on APTC. Twelve students participated. The mean professional experience was 15.5 years. The participants completed an informed consent form prior to the study. They completed a pre-course questionnaire, a post-course questionnaire, and a course evaluation form.

Results: The mean self-estimated improvement in theoretical knowledge pertaining to the course objectives was 8.23 on a 0 to 10 scale, and 8.25 for practical skills. Greatest improvement was in advanced airway management, physiological reactions to hypothermia, pneumothorax interventions, special considerations for patients injured by explosives (eg, blast injuries and burns), and medical decision making during an active shooter scenario. The evaluation of the simulation exercises received high marks (mean rating 4.53 [3.92-4.92] out of 5.0) on all aspects. The participants rated the overall course quality at 4.67 (on a 0 to 5 scale), with the simulations, practical exercises, and the structure of moving from theory to practice being mentioned as particularly positive.

Conclusion: Overall, the results showed that the APTC course received high marks on almost all measured factors. Further validation of the questionnaires is needed before general implementation of the battery can be recommended. Such implementation would benefit diverse course development and quality assurance.

Prehosp Disaster Med 2017;32(Suppl. 1):s222-s223

doi:10.1017/S1049023X17005763

An Electronic Competency-Based Evaluation Tool

for Assessing Humanitarian Competencies in a

Simulated Exercise

Andrea Evans ${ }^{1}$, Jennifer Hulme ${ }^{2}$, Hilarie Cranmer ${ }^{3}$,

Peter Nugus ${ }^{4}$, Melanie Coutu ${ }^{5}$, Kirsten N. Johnson ${ }^{6}$

1. Department Of Emergency Medicine, Hospital for Sick Children, Toronto/ON/Canada

2. Department Of Family Medicine, The University of Toronto, Toronto/AB/Canada

3. Global Disaster Response, Massachesetts General Hospital, Boston/MA/United States of America
4. Center For Medical Education, McGill University, Montreal/QC/ Canada

5. Humanitarian U, Montreal/QC/Canada

6. Department Of Family Medicine, McGill University, Montreal/ QC/Canada

Study/Objective: To present a novel, competency-based evaluation tool designed for rapid, electronic, offline use in a field-based simulation exercise.

Background: A growing number of humanitarian training programs are using simulation exercises in an effort to train and prepare humanitarians for work in the field. However, few field training exercises include methods and tools designed to assess the essential humanitarian competencies that participants must demonstrate in the SimEx and the field.

Methods: During a three-day humanitarian simulation event, participants in teams of eight to ten were individually evaluated at multiple injects by trained evaluators. Participants were assessed on five competencies and a global rating scale. Participants evaluated both themselves and their team members using the same tool at the end of the SimEx.

Results: All participants (63) were evaluated. A total of 1,008 individual evaluations were completed. There were 90 (9\%) missing evaluations. All 63 participants also evaluated themselves and each of their teammates using the same tool. Selfevaluation scores were significantly lower than peer-evaluations, which were significantly lower than evaluators' assessments. Participants with a medical degree, and those with humanitarian work experience of one month or more, scored significantly higher on all competencies assessed by evaluators compared to other participants. Participants with prior humanitarian experience scored higher on competencies regarding operating safely and working effectively as a team member.

Conclusion: This study presents a novel electronic evaluation tool to assess individual performance in five of six globally recognized humanitarian competency domains in a 3-day humanitarian SimEx. When combined with testing knowledge-based competencies, this presents an approach to a comprehensive competency-based assessment that provides an objective measurement of competency. There is an opportunity to advance the use of this tool in future humanitarian training exercises, and potentially in real time, in the field. This could impact the efficiency and effectiveness of humanitarian operations.

Prehosp Disaster Med 2017;32(Suppl. 1):s223

doi:10.1017/S1049023X17005775

\section{A Social Network Analysis of the Emergency Medical Command During a Live CBRNE Exercise Carl-Oscar Jonson ${ }^{1}$, Erik Prytz ${ }^{2}$, Ove Jansson ${ }^{1}$}

1. Centre For Teaching And Research In Disaster Medicine And Traumatology, And Department Of Clinical And Experimental Medicine, Linköping University, Linköping/Sweden

2. Department Of Computer And Information Science, Linköping University, Linköping/Sweden

Study/Objective: During major incidents, it is crucial that all actors in the emergency medical command have correct and 
current information. However, communication and situational awareness are often "named" problems at major incidents. This study used Social Network Analysis (SNA) to analyze emergency medical organizations' communication during a large-scale, mass-casualty Chemical, Biological, Radiological, and Nuclear (CBRN) exercise.

Background: Social Network Analysis (SNA) focuses on the relationship and resource (eg, information) sharing between actors in a given system. SNA can provide graphs of information transferred between actors and measure aspects such as sociometric status (how much "activity" an actor has in relation to others) and betweenness (how much interaction one actor has with all other actors).

Methods: The exercise scenario was a collision between a train carrying acrylonitrile and an excavator at a railroad crossing. Audio data were collected through microphones worn by the Ambulance Incident Commander (AIC) and the Medical Incident Commander (MIC), and also through radio recordings. The audio was transcribed, and meaningful utterances were entered into a social network matrix to produce social network statistics and graphical networks.

Results: The SNA showed that the four actors with the highest sociometric status were the MIC (11.83), AIC (9.97), RSC1 (4.66), and IC (2.59). The actors with highest betweenness were MIC (534.67), AIC (195.75), RSIC1 (47.25), and the train company representative from Veolia (5.00). A graphical representation is shown in Figure 1.

Conclusion: The SNA showed that the MIC and AIC had high information-sharing activity and interactions with other actors, as expected, given the organizational command structure. In Figure 1, it indicates several information-sharing structures, including pathways from higher command (DDO and Emergency Dispatch) to AIC, into a mostly interconnected network (bottom right) and several peripheral actors such as police officers. Overall, SNA appears to be a



Figure 1. Communication flow. useful tool to analyze communication during major CBRNE incidents.

Prehosp Disaster Med 2017;32(Suppl. 1):s223-s224

doi:10.1017/S1049023X17005787

System Dynamic Simulation for Medical Needs in the Great East Japan Earthquake

Shinichi Egawa ${ }^{1}$, Makoto Okumura ${ }^{2}$, Aya Murakami ${ }^{1}$, Tracey E. Clair Jones ${ }^{1}$, Hiroyuki Sasaki ${ }^{1}$

1. Division Of International Cooperation For Disaster Medicine, IRIDeS, Tohoku University, Sendai/Japan

2. Division Of Disaster Area Support, IRIDeS, Tohoku University, Sendai/Japan

Study/Objective: The aim of this study is to create a systemdynamics model simulating the medical needs in a disaster for the assessment of medical needs and decision making.

Background: In the Great East Japan Earthquake (GEJE) of 2011, the medical response team faced less patients with injury than expected, but an excess of patients with non-communicable disease (NCD), infectious disease, rehabilitation, mother and child health, and mental health needs in different time courses. It is crucial for the medical response team to predict the needs according to type of hazard, vulnerability, and capacity of the community. Besides precise analysis of real data, system dynamic simulation will enable us to postulate the dynamic change of inter-related medical needs in disaster.

Methods: Using Stella Architect software (Isee Systems, NH USA), the system dynamics model was built to represent each module of different medical needs. Japanese national average values of a crude birth rate $(8.8 / 1,000)$, inpatient $(1,090 /$ $100,000)$, outpatient $(5,376 / 100,000)$, and mental health problems (8.8/100) were used in a model of a given community (+65-old ratio is 32\%). Time-dependent ratio of injuries, locomotive syndromes, rehabilitation, mental health problems, $\mathrm{NCD}$, and infectious disease were assumed according to the experience of the GEJE. Time starts from 30 days before onset and goes through 365 days after onset.

Results: The model successfully simulated the time course of the total medical needs in a town devastated by GEJE, where all the health facilities were destroyed by a Tsunami. The simulation model does not include the relief capacities; the timedependent occurrence ratio was manually set. However, the total outcome became realistic and the relationship of various aspects of health needs in the time frame were visualized. Validation using the real medical needs data is necessary.

Conclusion: System dynamics model of medical needs in a disaster gives us a new insight in assessment and decision making. Prehosp Disaster Med 2017;32(Suppl. 1):s224

doi:10.1017/S1049023X17005799

A Comparison of PC Screen Based vs High Fidelity Simulation Supported Instruction in terms of Learning Outcomes and Cost

Kristine Qureshi

Nursing, University of Hawaii at Manoa, Honolulu/HI/United States of America 